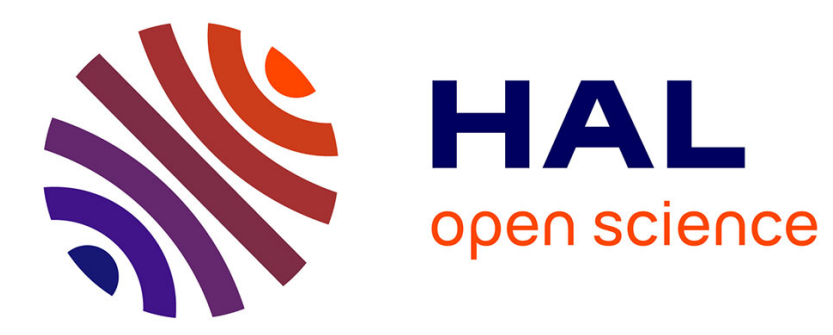

\title{
Diode-lasers : développements et applications
}

\author{
J. Yu
}

\section{To cite this version:}

J. Yu. Diode-lasers: développements et applications. Journal de Physique IV Proceedings, 1994, 04

(C4), pp.C4-610-C4-610. 10.1051/jp4:19944160 . jpa-00252617

\section{HAL Id: jpa-00252617 https://hal.science/jpa-00252617}

Submitted on 1 Jan 1994

HAL is a multi-disciplinary open access archive for the deposit and dissemination of scientific research documents, whether they are published or not. The documents may come from teaching and research institutions in France or abroad, or from public or private research centers.
L'archive ouverte pluridisciplinaire HAL, est destinée au dépôt et à la diffusion de documents scientifiques de niveau recherche, publiés ou non, émanant des établissements d'enseignement et de recherche français ou étrangers, des laboratoires publics ou privés. 


\section{Diode-lasers : développements et applications}

\section{J. YU}

Laboratoire Aimé Cotton, Campus d'Orsay, Bât. 505, 91405 Orsay cedex, France

Inventees en 1962, les diode-lasers de premiere gencration fonctionnant à la temperature de l'azote liquide, ont connu un début d'application difficile. Il a fallut attendre, au début des années 80 , le développement de la technologle de micro-électronique pour qu'enfin solent disponibles des diodes efficaces fonctionnant en temperature ambiante. Les applications en physique atomique et moléculaire prenaient l'essor, suscitant le développement de diodelaser de haute performance dans des laboratoires. Si les diodelasers représentaient une alternative aux lasers à colorant pour des expériences à budget modeste, Jeures performances actuelles montrent clairement la tendance: dans de nombreuses situations, elles peuvent compléter, remplacer, ou encore devancer les lasers á colorant dans des experiences qui exigent une grande quallte de lasers.

Dans notre laboratoire, nous avons developpe des diode-lasers monomodes, accordables et ulira stables, dont la largeur spectrale est réduite à mois de $100 \mathrm{kHz}$. Une telle performane est réalisée à partir de diodes commerciales dans les montages de couplage avec une cavite Fabry-Perot ou avec un reseau de diffraction. Dans certaines applications, la procédure d'injection optique à des diodes de puissance nous permettent d'obtenir jusqu'a $150 \mathrm{~mW}$ d'émission spectralement pure. Avec ces lasers stables et puissants, nous avons réalise des experiences originales dans le domaine des atomes froids et leures applications: ralentissement d'un jel atomique et obscrvation des franges de Ramesay sur le jet ralenti; mélasses optiques Doppler et. Sub-Doppler; compression d'un jet atomique monocinétlque dans un plége opto-magnetique; et transfert adiabatique de population par l'excitation de transition Raman.

Dans notre presentaion, nows nous rappelerons dabord, le principe de fonctionnement des diode-lasers ainsi que leures caracteristiques. Ensuitc, nous passerons en revue des principales techniques rendant une diode commerctale monomode et accordable avec une grande pureté spectralc. Nous présenterons enfin quelques exemples recents d'application de diode-lasers en physique atomique, en essayant de montrer leur rôle spěcifique. 